\title{
A Review on Variational Mode Decomposition for Rotating Machinery Diagnosis
}

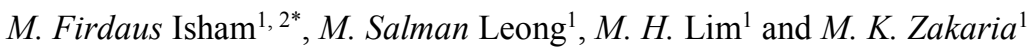 \\ ${ }^{1}$ Institute of Noise and Vibration, Universiti Teknologi Malaysia, Jalan Sultan Yahya Petra, 54100 Kuala Lumpur, Malaysia \\ ${ }^{2}$ Sekolah Kejuruteraan Mekanikal, Universiti Teknologi Malaysia, 81310 Skudai, Johor, Malaysia
}

\begin{abstract}
Signal processing method is very important in most diagnosis approach for rotating machinery due to non-linearity, non-stationary and noise signals. Recently, a new adaptive signal decomposition method has been proposed by Dragomiretskiy and Zosso known as variational mode decomposition (VMD). The VMD method has merit in solving mode mixing problem in most conventional signal decomposition method. This paper aims to review the applications of the VMD method in rotating machinery diagnosis. The advantages and limitations of the VMD method are discussed. Current solution on VMD limitation also have been review and discussed. Lastly, the future research suggestion has been pointed out in order to enhance the performance of the VMD method on rotating machinery diagnosis.
\end{abstract}

\section{Introduction}

Sudden failure is one of the most terrifying problem in most industrial application. It will caused losses in term of equipment and financial. Condition monitoring and fault diagnosis (CMFD) are the most common and effective approach used in many industries in order to avoid any sort of failures. There are a lots of CMFD approach have been studied and proposed [1-4].

Signal processing method is one of the most important element in CMFD. Empirical mode decomposition (EMD) is a popular signal processing method used in CMFD diagnosis studies [5-7]. However, recent study shows that this method suffer from mode mixing problem which makes a lots of EMD-improvised version have been proposed such as empirical EMD (EEMD) [8], complementary EEMD (CEEMD) [9], partial EEMD (PEEMD) [10], succinct and fast EMD (SF-EMD) [11], etc. Besides that, local mean decomposition (LMD) [12], intrinsic Time-scale decomposition (ITD) [13] and local characteristic-scale decomposition (LCD) [14] also have been proposed in order to solve the limitation of EMD. However, these method is not totally eliminate mode mixing problem $[15,16]$.

Recently, Dragomiretskiy and Zosso has proposed a new adaptive and modern signal analysis method called variational mode decomposition (VMD) [17]. The combination of Wiener filtering, Hilbert transform and alternate direction method of multiplier (ADMM) in VMD decomposition procedure give this method a huge merit in solving the mode mixing problem in most cases $[17,18]$. In recent years, the VMD method has been used in many application such as image processing [19], forecasting [20], speech recognition [21], machine monitoring [22], etc.

Therefore, this paper aims to review the application of the VMD method on rotating machinery diagnosis. This paper also aims to study the advantages and limitations of the VMD method in rotating machinery diagnosis for future improvements.

\section{Rotating Machinery Diagnosis}

The VMD method mainly used to decompose a signals into sets of sub-signals called as variational mode functions (VMFs). In rotating machinery application, it helps to separate a non-linear, non-stationary and noisy signals into sets of sub-signals according to the specific frequency range. Hence, the application of the VMD method in rotating machinery diagnosis is reviewed in this section according to different type of rotating machinery applications.

\subsection{Bearing Application}

In recent year, research on bearing application has gain a lots of interest from researchers with many bearing fault diagnosis approach has been proposed. The first VMD

\footnotetext{
* Corresponding author: firdaus060609@gmail.com
} 
application in bearing fault diagnosis study is done by Mohanty et al. where they using the VMD method to detect bearing fault at early stage [23]. A good comparison study between EMD and VMD also has been done by Mohanty et al. where they shows the superiority of the VMD method over EMD method [24]. Since then, it get a lots of attention from researcher to be further studied for bearing fault diagnosis.

Jinde et al. has proposed VMD, adaptive multiscale fuzzy entropy (AMFE) and support vector machine (SVM) for rolling bearing fault diagnosis [25]. Zhao and Li has proposed VMD and Teager energy operator (TEO) for wind turbine bearing diagnosis [26]. TEO is used to extract the fault features from selected VMFs. Li et al. also used VMD for bearing diagnosis by proposing independence-oriented VMD to identify bearing fault features [27]. Li et al. also proposed multi-dimensional VMD for bearing crack detection with large driving-speed variations [28]. Zhao and $\mathrm{Li}$ proposed wind turbine bearing diagnosis using VMD and spectrum kurtosis (SK) [29]. Tang et al. also study compound roller bearing diagnosis using blind source separation and VMD [30]. An et al. also study the wind turbine spherical roller bearing diagnosis using VMD and singular value decomposition (SVD) [31]. An et al. in another publication study wind turbine bearing diagnosis using VMD and permutation entropy [32]. Zhang et al. has study multistage centrifugal pump rolling bearing diagnosis using VMD [22]. Yi et al. proposed VMD based on particle swarm optimization (PSO) for rolling bearing fault diagnosis [33]. Zhu et al. also proposed VMD based on artificial fish swarm algorithm (AFSA) for rolling bearing diagnosis [34]. Table 1 summarize the literature on bearing diagnosis using VMD method.

Table 1: Bearing diagnosis based on VMD.

\begin{tabular}{|c|l|c|}
\hline $\begin{array}{c}\text { Diagnosis } \\
\text { Approach }\end{array}$ & \multicolumn{1}{|c|}{ Highlights } & Reference \\
\hline VMD, FFT & $\begin{array}{l}\bullet \text { Ball bearing } \\
\bullet \text { Vibration signals }\end{array}$ & {$[23]$} \\
\hline $\begin{array}{c}\text { VMD, AMFE, } \\
\text { SVM }\end{array}$ & $\begin{array}{l}\bullet \text { Rolling bearing } \\
\bullet \text { Vibration signals }\end{array}$ & {$[25]$} \\
\hline VMD, TEO & $\begin{array}{l}\bullet \text { Wind turbine } \\
\text { bearing }\end{array}$ & {$[26]$} \\
\hline MDVMD & $\begin{array}{l}\bullet \text { Vibration signals } \\
\text { bearing crack }\end{array}$ & {$[28]$} \\
\hline VMD, SK & $\begin{array}{l}\bullet \text { Vibration signals } \\
\text { bearing fault } \\
\bullet \text { Vibration signals }\end{array}$ & {$[29]$} \\
\hline BSS, VMD & $\begin{array}{l}\bullet \text { Roller bearing } \\
\bullet \text { Vibration signals }\end{array}$ & {$[30]$} \\
\hline VMD, SVD & $\begin{array}{l}\bullet \text { Wind turbine } \\
\text { spherical roller }\end{array}$ & {$[31]$} \\
& $\begin{array}{l}\text { bearing } \\
\text { Vibration signals }\end{array}$ & \\
\hline
\end{tabular}

\begin{tabular}{|c|l|c|}
\hline $\begin{array}{c}\text { VMD, } \\
\text { Envelope FFT }\end{array}$ & $\begin{array}{l}\bullet \text { Centrifugal pump } \\
\text { rolling bearing } \\
\bullet \text { Vibration signals }\end{array}$ & {$[22]$} \\
\hline VMD, PSO & $\begin{array}{l}\bullet \text { Rolling bearing } \\
\bullet \text { Vibration signals }\end{array}$ & {$[33]$} \\
\hline VMD, AFSA & $\begin{array}{l}\bullet \text { Rolling bearing } \\
\bullet \text { Vibration signals }\end{array}$ & {$[34]$} \\
\hline
\end{tabular}

\subsection{Gear Application}

Gear is another important application in rotating machinery as it also been used in many engineering applications. Gear diagnosis study also considered important is it has been study extensively in recent year. Muralidharan et al. started to use VMD in gear diagnosis study where they used VMD with random forest algorithm (RFA) to diagnose helical gear [35]. Xueli An et al. also used VMD together with envelope spectrum for gear diagnosis [36]. Mahgoun et al. study the capability of the VMD method in gear fault diagnosis under wariable speed condition [37]. Yan et al. proposed optimal VMD (OVMD) with 1.5-dimension envelope spectrum for gear fault diagnosis [38]. Zhang and Feng also study gear fault diagnosis by using VMD and demodulation analysis [39]. Feng et al. study planetary gearbox diagnosis using joint amplitude and frequency demodulation and VMD [40]. Firdaus et al. also used VMD and ELM for wind turbine gear diagnosis [18] . Li et al. also proposed VMD and deep neural networks (DNN) for planetary gear diagnosis [41]. Table 2 summarize the literature on gear diagnosis using VMD method.

Table 2: Gear diagnosis based on VMD.

\begin{tabular}{|c|c|c|}
\hline $\begin{array}{l}\text { Diagnosis } \\
\text { Approach }\end{array}$ & Highlights & Reference \\
\hline VMD, RFA & $\begin{array}{l}\text { - Helical gear } \\
\text { - Vibration signals }\end{array}$ & [35] \\
\hline $\begin{array}{l}\text { VMD, } \\
\text { Envelope } \\
\text { spectrum }\end{array}$ & $\begin{array}{l}\text { - Gear diagnosis } \\
\text { - Vibration signals }\end{array}$ & [36] \\
\hline VMD, FFT & $\begin{array}{l}\text { - Gear diagnosis } \\
\text { - Variable speed } \\
\text { - Vibration signals }\end{array}$ & [37] \\
\hline $\begin{array}{c}\text { OVMD, 1.5- } \\
\text { dimension } \\
\text { envelope } \\
\text { spectrum } \\
\end{array}$ & $\begin{array}{l}\text { - Gear diagnosis } \\
\text { - Vibration signals }\end{array}$ & [38] \\
\hline $\begin{array}{l}\text { VMD, } \\
\text { Demodulation } \\
\text { analysis }\end{array}$ & $\begin{array}{l}\text { - Gear diagnosis } \\
\text { - Vibration signals }\end{array}$ & [39] \\
\hline $\begin{array}{l}\text { VMD, Joint } \\
\text { Amplitude and } \\
\text { Frequency } \\
\text { demodulation }\end{array}$ & $\begin{array}{l}\text { - Planetary gear } \\
\text { diagnosis } \\
\text { - Vibration signals }\end{array}$ & [40] \\
\hline VMD, ELM & $\begin{array}{l}\text { - Wind turbine gear } \\
\text { diagnosis } \\
\text { - Vibration signals }\end{array}$ & [18] \\
\hline
\end{tabular}




\begin{tabular}{|l|l|l|}
\hline VMD, DNN & $\begin{array}{l}\bullet \text { Planetary gear } \\
\text { diagnosis } \\
\text { • Vibration signals }\end{array}$ & [41] \\
\hline
\end{tabular}

\subsection{Other Applications}

For other rotating machinery applications such as belt, shaft and blade are basically has less literature database as compared with gear and bearing. There is only one article found on shaft application using VMD and no article found for belt and blades using VMD methods. This has been studied by Jiang et al. where they using VMD and SVM for vertical-shaft load lifting monitoring [42].

\section{VMD Performance}

The VMD method has proof its capability to surpass the conventional EMD and its improvised version in most studies. This section will discussed the advantages and limitations of the VMD methods.

\subsection{Advantages}

The VMD method is not adopted a sifting process in its decomposition procedure which make this method has superiority in solving mode mixing problem. A great combination of Wiener filtering, Hilbert transform and ADMM makes the VMD method provide better and accurate decomposition result. Hence, the VMD method is able to decompose any signals into sets of VMFs accurately without eliminating useful information from the raw signals.

In order to present the superiority of the VMD method, three simulated signals has been used as shown in Equation 1-4 and Figure 1. Then, the simulated signals is decomposed using EMD and VMD method. Figure 2 and 3 shows the decomposition result for VMD and EMD for the $S(t)$.

$$
\begin{gathered}
s_{1}(t)=3 \sin (10 \pi t) \\
s_{1}(t)=0.4 \sin (400 \pi t) \\
s_{1}(t)=1.2 \sin (100 \pi t) \\
S(t)=s_{1}(t)+s_{2}(t)+s_{3}(t)
\end{gathered}
$$
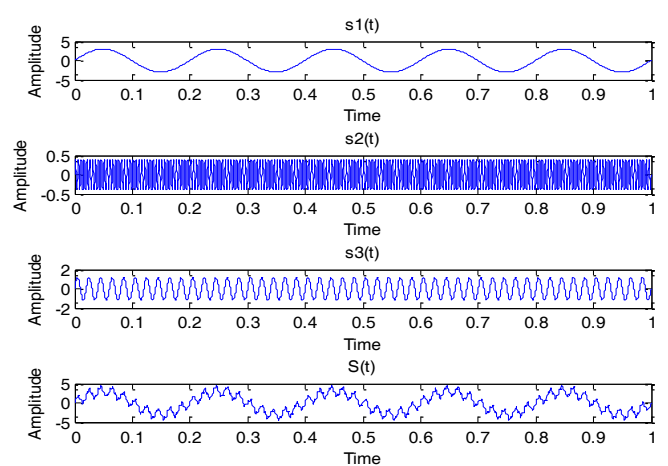

Figure 1: Simulated signals.

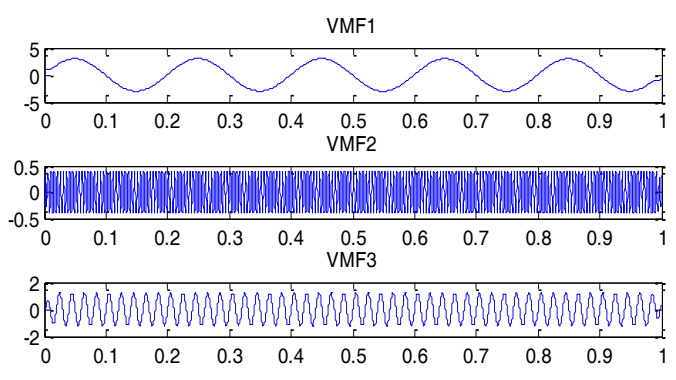

Figure 2: VMD decomposition result.

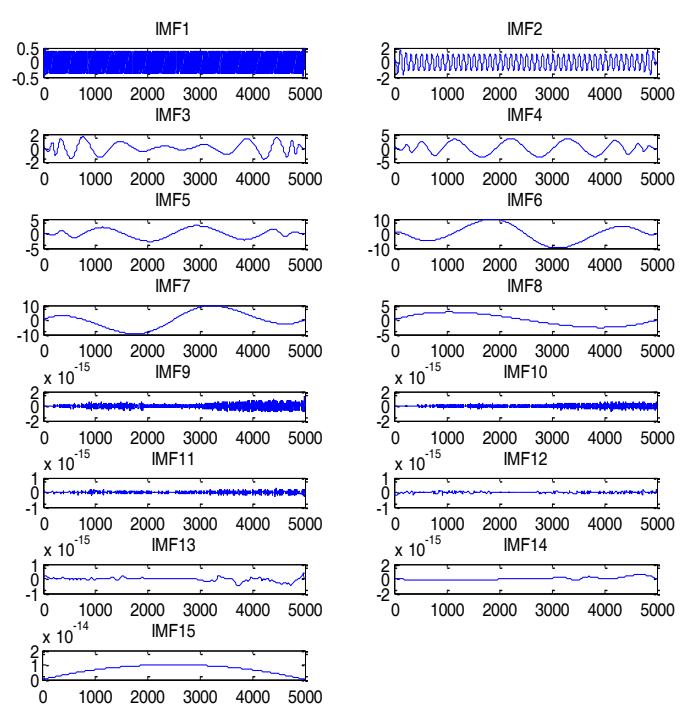

Figure 3: EMD decomposition result.

Based on these figures, it clearly shows that the VMD method is able to decompose $S(t)$ accurately as compared with the EMD method. There are a lots of mode mixing occurs in EMD decomposition result as shown in IMF3, IMF4, IMF5, IMF13 and IMF 14.

\subsection{Limitation}

The capability of the VMD method to decompose a signals accurately is totally depends on the settings of its input parameters. Basically, the VMD method consists of five main parameter which are mode number $(k)$, balancing parameter $(\alpha)$, time-step of dual ascent $(\tau)$, initial omega $(\omega)$ and tolerance $(\varepsilon)$. Some of these parameters has its standard value as suggested by Dragomiretskiy and Zosso. For an example, $\varepsilon$ is $1 \times$ $10^{-6}, \omega$ is 0 and $\tau$ is 0 for noise-slack. However, $k$ and $\alpha$ value are depends on the signal itself where different signals will have different values.

As shown in Figure 2, the $k$ and $\alpha$ values are set to 3 and 1500 . The $k$ value is 3 due to the number combination signals, $s_{n}(t)$. The $\alpha$ value is set to 1500 due to suggestion by Zhang et al. [22]. In rotating machinery diagnosis, the value for $k$ and $\alpha$ are unknown due lack of information and knowledge on the signal characteristic. Hence, 
developing a method to determine these parameter are crucial in order to ensure the performance of the VMD method. Setting inaccurate the value for $k$ and $\alpha$ will lead to over-decompose and under-decompose problem for the VMD method. Figure 4 and 5 shows the decomposition result when the $k$ value is set to 2 and 4 . Therefore, it is very important to have a good method to determine the value of $k$ and $\alpha$ for the VMD method.
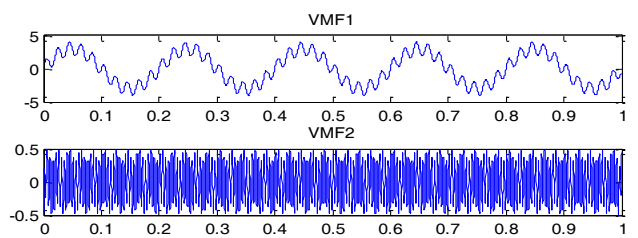

Figure 4: VMD decomposition with $k$ is 2.
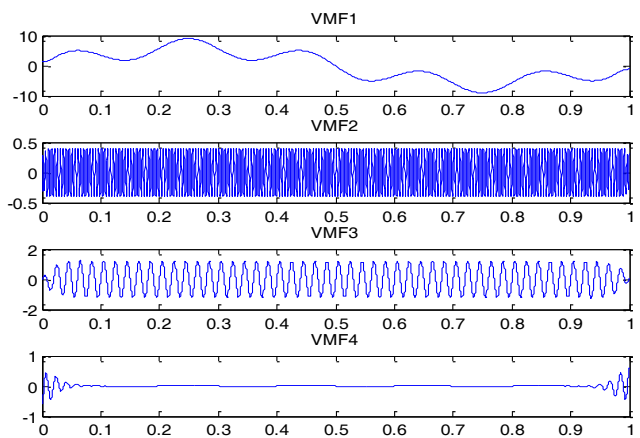

Figure 5: VMD decomposition with $k$ is 4 .
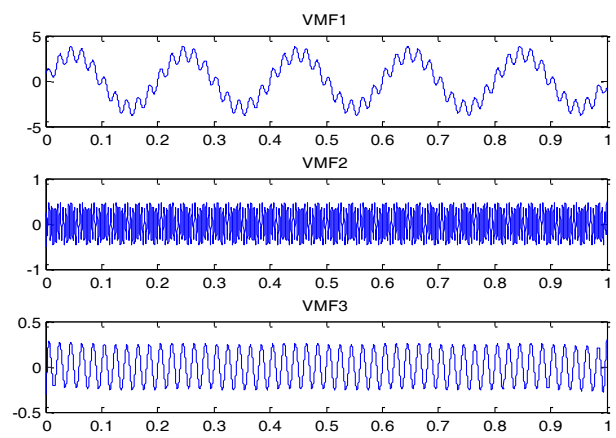

Figure 6 : VMD decomposition with $\alpha$ is 10 .
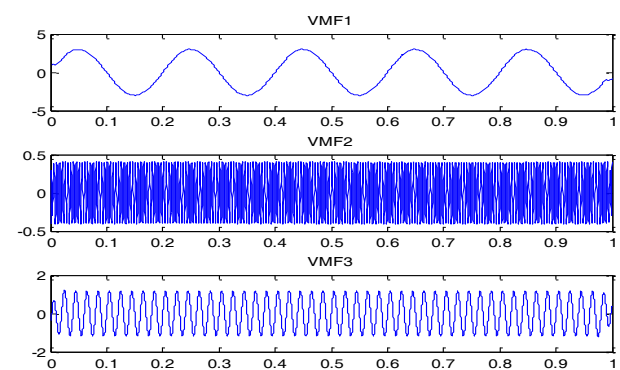

Figure 7 : VMD decomposition with $\alpha$ is 100 .

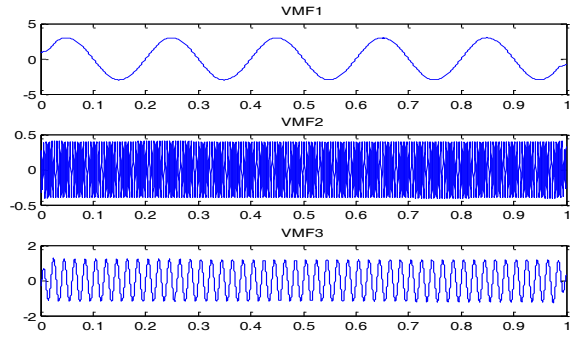

Figure 8: VMD decomposition with $\alpha$ is 1000 .
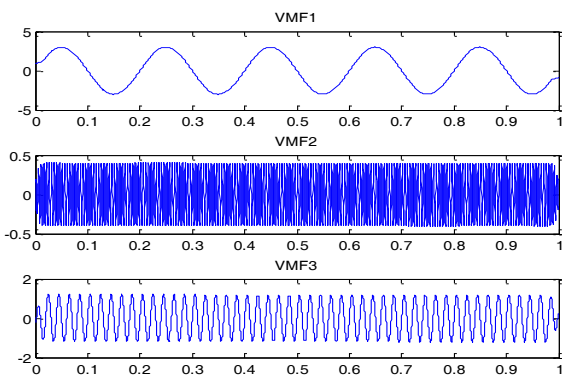

Figure 9 : VMD decomposition with $\alpha$ is 10000 .

Figure 4 shows the under-decompose problem for the VMD method and Figure 5 shows the over-decompose problem for the VMD method. Some modes are reconstructed accurately but some modes are not. Besides that, Figure 6-9 shows the decomposition result with different $\alpha$ value ranging from [10, 100, 1000, 10000$]$. It shows that the decomposition result only get stable when the $\alpha$ value is above 100 . Therefore, this simulation shows that the VMD method can only provide an accurate decomposition result when the setting of its input parameter is accurate.

\subsection{Current Solutions}

The limitation of the VMD method has gain a lots of attention from many researcher recently. In implementation of the VMD method in rotating machinery diagnosis, most researcher only concern on the $k$ value. The selection of the $k$ value is depends on the interest frequency for the study or it depends on the number of peaks from the frequency spectrum [36,37]. Recently, Li et al. proposed a stable and better selection method for $k$ using locally weighted scatterplot smoothing method (LOWESS) [27] Feng et al. also proposed a $k$ value selection based on spectral characteristic according to Equation 5 [40].

$$
k=\frac{F_{s}}{2 f_{m}}
$$

Where $F_{s}$ is sampling frequency and $f_{m}$ is gear meshing frequency. 
Then, a new approach for selecting the $k$ value is proposed using iterative procedure. Firdaus et al. has proposed an iterative selection method for $k$ using statistical parameter ratio (SPR) [18]. Zhang et al. also proposed an iterative method to select the $k$ value using correlation and energy ratio [22]. Yang et al. also proposed an iterative selection method for $k$ using cross correlation coefficient [43]. Besides that, some researcher also proposed a selection method using meta-heuristic algorithm to select the $k$ and $\alpha$ values. Yan et al. proposed a selection method using genetic algorithm (GA) [38]. Yi et al. proposed another selection method for $k$ and $\alpha$ using particle swarm optimization (PSO) [33]. Zhu et al. also proposed selection for $k$ and $\alpha$ using artificial fish swarm algorithm (AFSA) [34]. Ren et al. also proposed a selection method based on improve adaptive genetic algorithm (IAGA) [44]. Table 3 summarize the current solution for selecting $k$ and $\alpha$ values.

Table 3: Current solution to select $k$ and $\alpha$ values.

\begin{tabular}{|c|c|c|}
\hline Diagnosis Approach & $\begin{array}{c}\text { Selected } \\
\text { parameter }\end{array}$ & Reference \\
\hline LOWESS & - $k$ & {$[27]$} \\
\hline $\begin{array}{c}\text { Spectral characteristic } \\
\text { (Equation 5) }\end{array}$ & - $k$ & {$[40]$} \\
\hline SPR & - $k$ & [18] \\
\hline Correlation, energy ratio & $\begin{array}{ll}-k \\
\text { - } \alpha \\
\end{array}$ & {$[22]$} \\
\hline Cross correlation coefficient & - $k$ & {$[43]$} \\
\hline GA & $\begin{array}{l}\text { - } k \\
\text { - } \alpha\end{array}$ & {$[38]$} \\
\hline PSO & $\begin{array}{ll}-k \\
\text { - } \alpha \\
\end{array}$ & {$[33]$} \\
\hline AFSA & $\begin{array}{ll}-k \\
-\alpha \\
\end{array}$ & {$[34]$} \\
\hline IAGA & $\begin{array}{l}-k \\
\text { - } k\end{array}$ & [44] \\
\hline
\end{tabular}

\section{Conclusion and Future Remarks}

This paper review the application of the VMD method on rotating machinery diagnosis. The rotating machinery diagnosis involves are bearing, gears and shaft. Some rotating machinery diagnosis such as blade and belt still not using the VMD method for its diagnosis study. The advantages and limitations also have been presented. The VMD method provide better decomposition result as compared with the EMD. However, the selection of $k$ and $\alpha$ value is important to ensure the optimal performance of the VMD method. The current solution for selecting $k$ and $\alpha$ values also have been presented. Some of suggestion for future research gap can be summarized as follows:

1) The performance of the VMD method on blade and belt application is still unknown. A diagnosis study on these application will be useful for future benefits.

2) Latest meta-heuristic algorithm such as whale optimization algorithm (WOA) should be studied for selecting the $k$ and $\alpha$ values.

3) The computational efficiency is very important in rotating machinery diagnosis. The VMD method seems to use a lots of computational time to decompose a signals. Hence, an approach to reduce and minimize the computational time for the VMD method is a good research direction.

\section{Acknowledgement}

The authors would like to extend their greatest gratitude to the Institute of Noise and Vibration UTM for funding the study under the Higher Institution Centre of Excellence (HICoE) Grant Scheme (R.K130000.7809.4J226). Additional funding for this research also comes from the UTM Research University Grant (Q.K130000.2543.11H36), and Fundamental Research Grant Scheme (R.K130000.7840.4F653) by The Ministry of Higher Education Malaysia. Besides that, the author also want to acknowledge Eric Bechhoefer for providing the gearbox dataset from Vibration and Acoustic Database.

\section{References}

[1] Z. Li, Y. Jiang, C. Hu, Z. Peng, Recent progress on decoupling diagnosis of hybrid failures in gear transmission systems using vibration sensor signal: A review, Meas. J. Int. Meas. Confed. 90 (2016) 4-19.

doi:10.1016/j.measurement.2016.04.036.

[2] H. Zhao, M. Sun, W. Deng, X. Yang, A new feature extraction method based on EEMD and multi-scale fuzzy entropy for motor bearing, Entropy. 19 (2017). doi:10.3390/e19010014.

[3] X. Xue, J. Zhou, A hybrid fault diagnosis approach based on mixed-domain state features for rotating machinery, ISA Trans. (2016). doi:10.1016/j.isatra.2016.10.014.

[4] J. Lee, F. Wu, W. Zhao, M. Ghaffari, L. Liao, D. Siegel, Prognostics and health management design for rotary machinery systems - Reviews, methodology and applications, Mech. Syst. Signal Process. 42 (2014) 314-334. doi:10.1016/j.ymssp.2013.06.004.

[5] Y. Yu, YuDejie, C. Junsheng, A roller bearing fault diagnosis method based on EMD energy entropy and ANN, J. Sound Vib. 294 (2006) 269-277. doi:https://doi.org/10.1016/j.jsv.2005.11.002.

[6] J. Cheng, D. Yu, J. Tang, Y. Yang, Local rubimpact fault diagnosis of the rotor systems based on EMD, Mech. Mach. Theory. 44 (2009) 784- 
791.

doi:10.1016/j.mechmachtheory.2008.04.006.

[7] C. Junsheng, Y. Dejie, Y. Yu, A fault diagnosis approach for roller bearings based on EMD method and AR model, Mech. Syst. Signal Process. 20 (2006) 350-362. doi:10.1016/j.ymssp.2004.11.002.

[8] N.E. Huang, Z. Wu, Ensemble Empirical Mode Decomposition: a Noise-Assisted Data Analysis Method, Adv. Adapt. Data Anal. 01 (2009) 1. doi:10.1142/S1793536909000047.

[9] J.-R. Yeh, J.-S. Shieh, Complementary Ensemble Empirical Mode Decomposition: A Novel Enhanced Data Analysis Method, Adv. Adapt. Data Anal. 2 (2010) 135-156. doi:10.1142/S1793536910000422.

[10] J. Zheng, J. Cheng, Y. Yang, Partly ensemble empirical mode decomposition: An improved noise-assisted method for eliminating mode mixing, Signal Processing. 96 (2014) 362-374. doi:10.1016/j.sigpro.2013.09.013.

[11] H. Li, Y. Hu, F. Li, G. Meng, Succinct and fast empirical mode decomposition, Mech. Syst. Signal Process. 85 (2017) 879-895. doi:10.1016/j.ymssp.2016.09.031.

[12] J.S. Smith, The local mean decomposition and its application to EEG perception data, J. R. Soc. Interface. 2 (2005) 443-454. doi:10.1098/rsif.2005.0058.

[13] M.G. Frei, I. Osorio, Intrinsic time-scale decomposition : time - frequency - energy analysis and real-time filtering of non-stationary signals, Proc. R. Soc. A. (2007) 321-342. doi:10.1098/rspa.2006.1761.

[14] J. Cheng, Y. Yang, Y. Yang, Local characteristic-scale decomposition method and its application to gear fault diagnosis, Jixie Gongcheng Xuebao/Journal Mech. Eng. 48 (2012) 64-71. doi:10.3901/JME.2012.09.064.

[15] Y. Li, M. Xu, Y. Wei, W. Huang, Rotating machine fault diagnosis based on intrinsic characteristic-scale decomposition, Mamt. 94 (2015) 9-27.

doi:10.1016/j.mechmachtheory.2015.08.001.

[16] A. Hu, X. Yan, L. Xiang, A new wind turbine fault diagnosis method based on ensemble intrinsic time-scale decomposition and WPTfractal dimension, Renew. Energy. 83 (2015) 767-778. doi:10.1016/j.renene.2015.04.063.

[17] K. Dragomiretskiy, D. Zosso, Variational Mode Decomposition, IEEE Trans. Signal Process. 62 (2014) 531-544. doi:10.1109/tsp.2013.2288675.

[18] M.F. Isham, M.S. Leong, M.H. Lim, Z.A. Ahmad, Variational Mode Decomposition for Rotating Machinery Condition Monitoring Using Vibration Signals, Trans. Nanjing Univ. Aero. Astro. 35 (2018) 38-50. doi:10.16356/j.10051120.2018.01.038.
[19] S. Lahmiri, A. Shmuel, Variational mode decomposition based approach for accurate classification of color fundus images with hemorrhages, Opt. Laser Technol. 96 (2017) 243-248.

doi:http://dx.doi.org/10.1016/j.optlastec.2017.05. 012.

[20] S. Lahmiri, Intraday stock price forecasting based on variational mode decomposition, J. Comput. Sci. 12 (2016) 23-27. doi:https://doi.org/10.1016/j.jocs.2015.11.011.

[21] A. Upadhyay, R.B. Pachori, Instantaneous voiced/non-voiced detection in speech signals based on variational mode decomposition, J. Franklin Inst. 352 (2015) 2679-2707. doi:https://doi.org/10.1016/j.jfranklin.2015.04.00 1.

[22] M. Zhang, Z. Jiang, K. Feng, Research on variational mode decomposition in rolling bearings fault diagnosis of the multistage centrifugal pump, Mech. Syst. Signal Process. 93 (2017) 460-493. doi:https://doi.org/10.1016/j.ymssp.2017.02.013.

[23] Mohanty, K.K. Gupta, K.S. Raju, Bearing fault analysis using variational mode decomposition, in: 2014 9th Int. Conf. Ind. Inf. Syst., 2014: pp. 1-6. doi:10.1109/ICIINFS.2014.7036617.

[24] S. Mohanty, K.K. Gupta, K.S. Raju, Comparative study between VMD and EMD in bearing fault diagnosis, in: 2014 9th Int. Conf. Ind. Inf. Syst., 2014: pp. 1-6. doi:10.1109/ICIINFS.2014.7036515

[25] Z. Jinde, J. Zhanwei, P. Ziwei, Z. Kang, VMD based adaptive multiscale fuzzy entropy and its application to rolling bearing fault diagnosis, Proc. Int. Conf. Sens. Technol. ICST. (2016) 03. doi:10.1109/ICSensT.2016.7796267.

[26] H. Zhao, L. Li, Fault diagnosis of wind turbine bearing based on variational mode decomposition and Teager energy operator, IET Renew. Power Gener. 11 (2016) 453-460. doi:10.1049/iet-rpg.2016.0070.

[27] Z. Li, J. Chen, Y. Zi, J. Pan, Independenceoriented VMD to identify fault feature for wheel set bearing fault diagnosis of high speed locomotive, Mech. Syst. Signal Process. 85 (2017) 512-529. doi:10.1016/j.ymssp.2016.08.042.

[28] Z. Li, Y. Jiang, Q. Guo, C. Hu, Z. Peng, Multidimensional variational mode decomposition for bearing-crack detection in wind turbines with large driving-speed variations, Renew. Energy. (n.d.). doi:https://doi.org/10.1016/j.renene.2016.12.013.

[29] H. Zhao, L. Li, Fault diagnosis of wind turbine bearing based on variational mode decomposition and Spectrum Kurtosis, IET Renew. Power Gener. (2016) 851-854. doi:10.1049/iet-rpg.2016.0070. 
[30] G. Tang, G. Luo, W. Zhang, C. Yang, H. Wang, Underdetermined blind source separation with variational mode decomposition for compound roller bearing fault signals, Sensors

(Switzerland). 16 (2016). doi:10.3390/s16060897.

[31] X. An, H. Zeng, Fault diagnosis method for spherical roller bearing of wind turbine based on variational mode decomposition and singular value decomposition., J. Vibroengineering. 18 (2016) 3548-3556. http://10.0.84.91/jve.2016.16553.

[32] X. An, L. Pan, Bearing fault diagnosis of a wind turbine based on variational mode decomposition and permutation entropy, Proc. Inst. Mech. Eng. Part O J. Risk Reliab. 231 (2017) 200-206. doi:10.1177/1748006X17693492.

[33] C. Yi, Y. Lv, Z. Dang, A fault diagnosis scheme for rolling bearing based on particle swarm optimization in variational mode decomposition, Shock Vib. 2016 (2016). doi:10.1155/2016/9372691.

[34] J. Zhu, C. Wang, Z. Hu, F. Kong, X. Liu, Adaptive variational mode decomposition based on artificial fish swarm algorithm for fault diagnosis of rolling bearings, Proc. Inst. Mech. Eng. Part C J. Mech. Eng. Sci. 231 (2017) 635654. doi:10.1177/0954406215623311.

[35] A. Muralidharan, V. Sugumaran, K.P. Soman, M. Amarnath, Fault diagnosis of helical gear box using variational mode decomposition and random forest algorithm, SDHM Struct. Durab. Heal. Monit. 10 (2015) 55-80.

http://www.scopus.com/inward/record.url?eid=2 -s2.0-84926337674\&partnerID=tZOtx3y1.

[36] X. An, H. Zeng, C. Li, Envelope demodulation based on variational mode decomposition for gear fault diagnosis, Proc. Inst. Mech. Eng. Part E J. Process Mech. Eng. (2016) 0954408916644271. doi:10.1177/0954408916644271.

[37] H. Mahgoun, F. Chaari, A. Felkaoui, Detection of gear faults in variable rotating speed using variational mode decomposition (VMD), Mech. Ind. 17 (2016).

https://doi.org/10.1051/meca/2015058.

[38] X. Yan, M. Jia, L. Xiang, Compound fault diagnosis of rotating machinery based on OVMD and a 1.5-dimension envelope spectrum, Meas. Sci. Technol. 27 (2016) 75002.

http://stacks.iop.org/0957$0233 / 27 / \mathrm{i}=7 / \mathrm{a}=075002$.

[39] D. Zhang, Z. Feng, Application of variational mode decomposition based demodulation Analysis in gearbox fault diagnosis, Conf. Rec. IEEE Instrum. Meas. Technol. Conf. 2016-July (2016). doi:10.1109/I2MTC.2016.7520586.

[40] Z. Feng, D. Zhang, M. Zuo, Planetary Gearbox Fault diagnosis via Joint Amplitude and
Frequency Demodulation Analysis Based on Variational Mode Decomposition, Appl. Sci. 7 (2017) 775. doi:10.3390/app7080775.

[41] Y. Li, G. Cheng, C. Liu, X. Chen, Study on planetary gear fault diagnosis based on variational mode decomposition and deep neural networks, Meas. J. Int. Meas. Confed. 130 (2018) 94-104. doi:10.1016/j.measurement.2018.08.002.

[42] F. Jiang, Z. Zhu, W. Li, S. Xia, G. Zhou, Lifting load monitoring of mine hoist through vibration signal analysis with variational mode decomposition, J. Vibroengineering. 19 (2017) 6021-6035.

[43] H. Yang, S. Liu, H. Zhang, Adaptive estimation of VMD modes number based on cross correlation coefficient, J. Vibroengineering. 19 (2017) 1185-1196.

[44] G. Ren, J. Jia, J. Mei, X. Jia, J. Han, Y. Wang, An improved variational mode decomposition method and its application in diesel engine fault diagnosis, J. Vibroengineering. 20 (2018) 23632378 . 